\title{
Cerebral Coagulation Complications Following COVID-19 Adenoviral Vector Vaccines: A Systematic Review
}

\author{
Sara Aghabaklou ${ }^{\mathrm{a}}$, Seyed-Mostafa Razavi ${ }^{\mathrm{b}}$, Pegah Mohammadic, \\ Sharareh Gholamin ${ }^{\mathrm{d}}$, Ashkan Mowla ${ }^{\mathrm{e}, \mathrm{f}}$
}

\begin{abstract}
Emergence of the novel coronavirus, severe acute respiratory syndrome coronavirus 2 (SARS-CoV-2) outbreak identified in late 2019 in Wuhan, China, was declared a pandemic in March 2020. High fatality rate in afflicted patients prompted scientists and physicians to develop various vaccines against the virus. While administration of millions of doses of the adenoviral vector vaccines (e.g., Oxford-AstraZeneca (ChAdOx1 nCoV-19) and Janssen/Johnson \& Johnson (Ad26.COV2. S)) has helped control the disease, numerous cases of cerebral venous sinus thrombosis (CVST) with thrombocytopenia have been reported in vaccinated individuals. In this article, we aim to review the cases reported thus far and further discuss the association between the vaccine administration and subsequent cerebral thromboembolic events. Our study was performed and reported based on the guidelines outlined in the Preferred Reporting Items for Systematic Reviews and Meta-Analyses (PRISMA). PubMed, Google Scholar and Norris Medical Library databases were searched using the following terms: coronavirus disease 2019 (COVID-19) vaccines ("AstraZeneca" or "AZD1222 COVID vaccine" or "ChAdOx1 nCoV-19 COVID-19 vaccine" or "Janssen" or "Johnson \& Johnson COVID vaccine" or "Ad26.COV2 COVID vaccine"), coagulopathy ("cerebral venous sinus thrombosis (CVST)" and "vaccine-induced immune thrombotic thrombocytopenia (VITT)" or "cerebral venous thrombosis (CVT)") and thrombocytopenia. All the relevant studies within the English literature up to August 1, 2021, were included. Fourteen most recent articles reporting on 66 patients with CVST and VITT after adenoviral vector vaccination were reviewed by two independent authors. Age of the patients ranged from 18 to 60 years. The majority
\end{abstract}

Manuscript submitted July 26, 2021, accepted September 2, 2021

Published online October 5, 2021

${ }^{a}$ Department of Preventive Medicine, Keck School of Medicine, University of Southern California, Los Angeles, CA, USA

${ }^{b}$ Heart and Rhythm Clinic, San Jose, CA, USA

'Department of Medicine, Eisenhower Medical Center, Rancho Mirage, CA, USA

${ }^{\mathrm{d}}$ Department of Biology and Bioengineering at California Institute of Technology, Pasadena, CA, USA

eDepartment of Neurological Surgery, Keck School of Medicine, University of Southern California, Los Angeles, CA, USA

${ }^{f}$ Corresponding Author: Ashkan Mowla, Division of Endovascular Neurosurgery, Department of Neurological Surgery, Keck School of Medicine, University of Southern California (USC), Suite 3300, 1200 North State St., Los Angeles, CA 90033, USA. Email: mowla@usc.edu of cases were women (43 females versus 14 males). Platelet count was between 5 and $127 \times 109 /$ L. Above-normal D-dimer was found in $86 \%$ of the patients. A total of $68 \%$ of the patients had positive platelet factor $4 \mathrm{IgG}$ assay in the absence of prior exposure to heparin. Among CVST cases following COVID vaccination, $44 \%$ succumbed to death. Early diagnosis and treatment of CVST plays a fundamental role in decreasing morbidity and mortality. Health care professional should be familiar with this rare complication post vaccination against COVID-19. Given the rarity of CVST after the COVID-19 vaccine, the benefit of vaccination outweighs the potential harm.

Keywords: COVID-19; Adenoviral vector vaccines; AstraZeneca; AZD1222 COVID vaccine; Johnson \& Johnson COVID vaccine; Coagulopathy; Thrombocytopenia; Cerebral venous sinus thrombosis

\section{Introduction}

\section{Coronavirus disease 2019 (COVID-19) virus}

The novel coronavirus, severe acute respiratory syndrome coronavirus 2 (SARS-CoV-2), identified in late 2019 in Wuhan, China, spread rapidly around the globe and was announced a pandemic on March 11, 2020 [1]. The single stranded positive sense ribonucleic acid (RNA) virus, SARS-CoV-2 [2, 3] spreads through droplet and is associated with a wide range of clinical complications, from fever, cough, muscle or body aches, loss of taste or smell to more severe consequences such as pneumonia, organ failure, acute respiratory distress syndrome, acute kidney injury, and thromboembolic events (e.g., venous thromboembolism, pulmonary embolism, deep vein thrombosis, stroke, myocardial infarction, and limb artery thrombosis) [4]. Severe clinical complications have been found in more advanced cases, especially in intensive care unit patients. The underlying mechanism through which SARSCoV-2 exerts its effect on different organs of the human body is not well understood; however, aftermaths of cytokine storm and thromboembolic associated disorders are the main culprits in developing fatality and long-lasting disorders [2, 5-7].

\section{Vaccines}

After significant efforts across the world to develop effective 
vaccines and therapeutic modalities, two categories of vaccines have been emerged with promising response against the coronavirus that encode the spike protein of SARS-CoV-2: mRNAbased vaccines such as BNT162b2 (Pfizer-BioNTech) and mRNA-1273 (Moderna), and adenoviral vector vaccines such as ChAdOx1 nCoV-19 (Vaxzevria, previously Oxford-AstraZeneca) and Ad26.COV2.S (Janssen-Johnson \& Johnson) [8, 9].

\section{Vaccine complications}

More than 2 billion doses of the vaccines against COVID-19 were administered worldwide by June2021, resulting in reduced number of severe cases and mortality [10]. Similar to other vaccines, the COVID-19 vaccine can cause benign complications such as headache, fever, diarrhea, and pain at the injection site. Aside from these benign symptoms, days after starting vaccination with Vaxzevria, and at the same time after Janssen-Johnson \& Johnson vaccination, numerous cases of thromboembolic events, e.g., cerebral venous sinus thrombosis (CVST) with low platelet counts were reported, particularly in Europe [8, 10-12].

On April 4, 2021, European Medicines Agency (EMA)'s safety committee Pandemic Response Accountability Committee announced that 169 cases of CVST and 53 cases of superficial vein thrombosis have been reported after administration of the Oxford-AstraZeneca vaccine [13]. On April 7, 2021, EMA Pharmacovigilance Risk Assessment Committee (PRAC) stated that there may be a relationship between vaccination with Vaxzevria and thrombosis with thrombocytopenia (TTS) within the cerebral venous sinus [10]. The US Food and Drug Administration (FDA) recommended a temporary pause in the use of the Janssen-Johnson \& Johnson vaccines on April 12, 2021, after six cases of CVST with thrombocytopenia were pointed out within approximately 7 million vaccinated individuals in the USA [10, 14]. After reviewing the data on TTS cases, the Advisory Committee on Immunization Practices of Centers for Disease Control and Prevention (CDC) recommended that the Johnson \& Johnson vaccination can be reinstated $[11,14]$. European countries also resumed Vaxzevria vaccination, although some of these countries restricted its use by age [10].

Thrombosis associated with thrombocytopenia after vaccination has been named vaccine-induced prothrombotic immune thrombocytopenia or vaccine-induced immune thrombotic thrombocytopenia (VITT), collectively designated as VITT in the current review. Thromboembolic events occurred 5 - 21 days after COVID-19 vaccine administration in unusual sites such as the abdomen and brain and can vary widely from deep vein thrombosis to pulmonary embolism, splanchnic, portal or hepatic vein thrombosis, CVST, and ophthalmic vein thrombosis $[15,16]$.

The association between vaccine administration and the subsequent thromboembolic event is not well understood. To this end, here in this review, we summarized 23 articles that explicitly discussed cerebral vaccination-induced coagulopathy with associated thrombocytopenia. Our focus relies on understanding the clinical and laboratory findings in postCOVID-19 vaccination cerebral coagulopathies and providing new insight about the patients at risk of developing thrombo- embolic diseases following vaccination.

\section{Methods}

Our study was performed and reported based on the guidelines outlined in the Preferred Reporting Items for Systematic Reviews and Meta-Analyses (PRISMA, Fig. 1). A comprehensive literature search was performed on PubMed, Google Scholar and Norris Medical Library databases using the following key terms: COVID-19vaccines ("AstraZeneca" or "AZD1222 COVID vaccine" or "ChAdOx1 nCoV-19 COVID-19 vaccine" or "Janssen" or "Johnson \& Johnson COVID vaccine" or "Ad26.COV2 COVID vaccine"), coagulopathy ("cerebral venous sinus thrombosis (CVST)" and "vaccineinduced immune thrombotic thrombocytopenia (VITT)" or "cerebral venous thrombosis (CVT)") and thrombocytopenia. All the relevant articles on post-vaccine CVST and associated VITT in humans presenting at least one case of CVST after the COVID-19 vaccination within the English literature until August 1, 2021, were included. The articles were reviewed by two independent authors and the data were entered into a data sheet. Protocols without findings, articles without CVST cases and articles published in a language other than English were excluded from the current systematic review.

\section{Findings}

This review surveyed 23 most recent articles reporting on 66 patients with CVST and associated VITT following vaccination against COVID-19 [5, 14-35]. The reviewed papers are summarized here (Supplementary Material 1, www.neurores. org) with focus on functional outcome, characteristics of the patients, clinical manifestations and imaging and laboratory findings. The age of the patients was $>18$ (range: $18-60$ ) years old. The majority of cases were seen among women (43 females versus 14 males). Platelet count in patients was between 5 and $127 \times 10^{9} / \mathrm{L}$. Most of the patients lacked a significant past medical history. Two patients had hypothyroidism and two had Hashimoto's thyroiditis. None of the patients had active cancer or had used any anticoagulation before admission. Two of the patients were on antihypertensive medications, three had a recent history of oral contraceptive use, one had the contraceptive vaginal ring, one case was on hormone replacement therapy, one patient had a history of type 1 von Willebrand disease and positive anticardiolipin antibody, and one patient had prothrombin mutation. Obesity was reported in six patients. The international normalized ratio (INR) was normal in almost all of the patients. Ninety-six percent of the patients had low platelet levels and $86 \%$ had increased D-dimer. A total of $68 \%$ of the patients had positive platelet factor 4 (PF4) IgG assay in the absence of prior exposure to heparin.

CVST associated with VITT following adenoviral vector vaccination is a rare syndrome that occurs approximately 5 - 19 days following vaccination. Although rare, the mortality rate has been reported as high as $39 \%$. The most common 


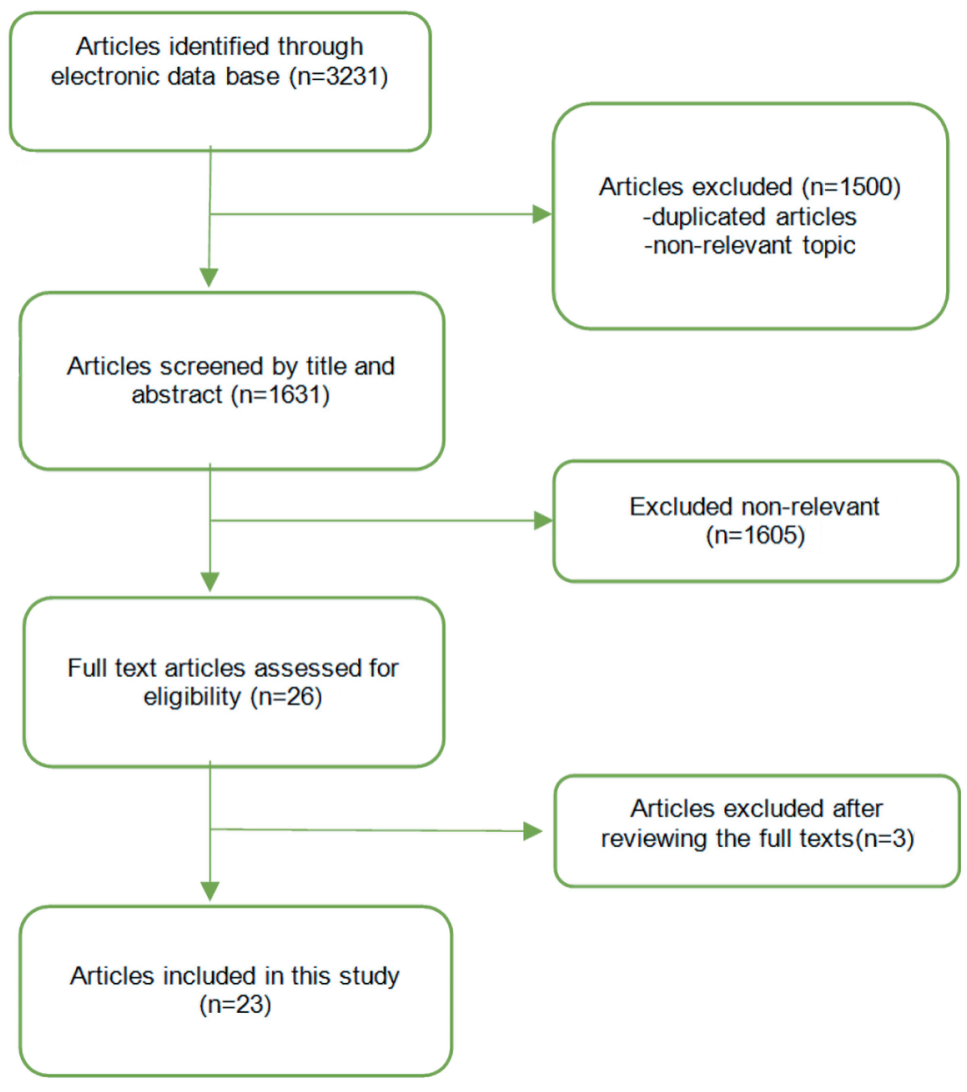

Figure 1. Study identification and selection process.

clinical presentations of the patients with CVST and thrombocytopenia following adenoviral vector vaccination are similar to typical CVST patients. The majority of the patients in this review presented initially with headaches. Per the American Heart Association recommendations, persistent headaches should increase concern for CVST or other thrombotic events post adenoviral vector vaccination [36].

\section{Discussion}

Knowing the mechanism of hypercoagulability induced by SARS-CoV-2 infection may help researchers elaborate further on post-COVID-19 vaccination-associated VITT [37]. Coronavirus may also increase the risk of coagulation through other unknown pathophysiological mechanisms [38, 39]. The mechanism of coagulation by COVID-19 is initiated by the binding of spike surface protein of SARS-CoV-2 to human angiotensin-converting enzyme (ACE) 2 receptor, causing direct viral endothelial invasion, endothelial apoptosis, and neuronal damage [38, 40-54]. Studies have found that infection with SARS-CoV-2 and activation of the coagulation cascade, cytokine storm-related prothrombotic state, and/or increased platelet activity can accelerate the progression of cerebrovascular events including CVST $[6,40,41]$. CVST has been reported as a very rare neurological complication of COVID-19 patients during the pandemic [55].
Mainly seen in women of childbearing age, CVST is often difficult to diagnose due to heterogeneous and non-specific neurological manifestations such as headache, seizure, altered consciousness, and focal neurological signs [56, 57]. Most CVST patients (73-82.6\%) have relevant risk factors [56] including thrombophilia, oral contraceptives, fasting, malignancy, infection, trauma, pregnancy, puerperium [58-61], lumbar puncture, neurosurgical procedures, tumor compression, and endocrine disturbances $[31,62]$.

With more doses of adenoviral vector vaccine given each day, numerous cases of CVST with thrombocytopenia have been reported in vaccinated individuals. The symptoms that triggered seeking medical attention in post-COVID-19 vaccinated CVST patients were similar to other CVST cases. To establish the diagnosis of CVST associated with VITT after COVID-19 vaccination in patients with neurological symptoms, laboratory evidence should be present along with a diagnosis of CVST on imaging modalities [38]. Laboratory features in post-COVID-19 vaccinated CVST patients were platelet count below $100 \times$ $10^{9} / \mathrm{L}$, high D-dimer, and low serum fibrinogen [5]. CVST was assessed via non-invasive imaging such as brain magnetic resonance imaging (MRI), magnetic resonance venography (MRV), and computed tomography venography (CTV) [38].

Immunologic interactions and coagulopathy have been previously reported with other vaccines [63]. In 1973, thrombotic thrombocytopenia was reported post influenza vaccination [64]. Reports of similar event after H1N1, rabies and 
pneumococcal vaccination also exist [65-68]. Nevertheless, CVST has not been reported in any of these cases $[64,66]$.

Vaxzevria and Janssen-Johnson \& Johnson vaccines contain replication-incompetent adenoviral vectors from chimpanzees and humans, respectively. Both recombinant factors encode the spike glycoprotein of SARS-CoV-2 which may lead to activation of immune cells and coagulopathy [69, 70]. Rare cases of extensive thrombosis associated with severe thrombocytopenia and disseminated intravascular coagulation along with the presence of anti-PF4 antibodies have been reported after the AstraZeneca vaccine [2, 29].

The presence of anti-PF4 antibodies in post-COVID-19 vaccine CVST and VITT cases points out to an underlying immunologic phenomenon predisposing to platelet consumption, thrombocytopenia and thrombosis; a mechanism similar to heparin-induced thrombocytopenia (HIT), fittingly named spontaneous [71] or autoimmune HIT syndrome [22, 72].

Classic HIT is caused by antibodies against heparin/platelet PF4 complexes [73] and occurs in $0.76 \%$ of hospitalized patients receiving intravenous thromboprophylaxis [74].

In contrast to the classic HIT, in which immune activation is caused by exogenous heparin, in spontaneous (autoimmune) HIT an endogenous anion such as polyphosphate or chondroitin sulfate causes anti-PF4 antibody formation [14, 72]. It has been proposed that part of the adenoviral vector vaccine cross-reacts with PF4 on platelets due to molecular mimicry, causing anti-PF4 antibody formation (antibody responses against PF4/polyanion) and may play role in VITT syndrome cases after AstraZeneca COVID-19 vaccine administration [72, 75, 76]. Researchers have hypothesized that complement activation by antibodies against vaccine components can stimulate VITT [77]. Similar to heparin's "vaccine-induced COVID-19 mimicry," production of the soluble spike protein variant encoded by adenoviral vector vaccine acts as a polyanion to mediate PF4 activation and trigger antibody-dependent cell-mediated cytotoxicity (ADCC) or complement-dependent cytotoxicity $[10,78]$.

Nonetheless, adenovirus interaction with platelets in vivo has been reported to deplete platelets [79]. Moreover, continuous tissue compression in major surgeries can induce anti-PF4/ polyanion antibodies without heparin treatment when mechanical compression devices are used for thrombosis prophylaxis [10]. Tissue micro-trauma and deoxyribonucleic acid (DNA) release from the vaccine promote adenoviral contact with PF4 and increase anti-PF4 autoantibodies $[10,76]$. Cluster of differentiation (CD)147 has been associated with thrombosis in SARS-CoV and human immunodeficiency virus (HIV)-1 but no direct role for SARS-CoV-2 has been identified yet [76]. Thrombocytopenia is the most common laboratory finding in HIT [73], a phenomenon also observed in the 66 patients studied here who received the COVID-19 vaccine and developed CVST associated with VITT.

\section{Treatment}

In suspected CVST patients associated with VITT who are also positive foranti-PF4 antibody, the recommended treatment is similar to that of severe HIT. Due to the similarity of pathophysiology and laboratory findings of post-COVID vac- cination VITT-associated CVST to HIT syndrome, heparin avoidance could be considered in the treatment plan [80]. Collaboration among vascular neurologists, hematologists, and neurosurgeons consultants for managing systemic thrombosis is very important.

Anticoagulant therapy can be achieved with non-heparin agents [81], e.g., direct thrombin inhibitors (argatroban and bivalirudin), direct factor Xa inhibitors (rivaroxaban and apixaban), fondaparinux, and danaparoid [80, 82, 83] chosen according to the patient's clinical status (risk of bleeding or need for an invasive procedure) [84].

Despite very sparse data, administration of intravenous immunoglobulin (IVIG) ( $1 \mathrm{~g} / \mathrm{kg}$ body weight) daily for 2 days has been suggested after anti-PF4 antibodies have been sent [80].

If imaging does not show overt thrombosis, but thrombocytopenia with D-dimer elevation is present, thromboprophylaxis with non-heparin anticoagulants should be started [84].

Moreover, in CVST patients with an impending herniation neurosurgical intervention may be necessary with decompressive craniotomy [31]. Platelet transfusion should be avoided in CVST patients associated with VITT who are also positive for anti-PF4 antibody due to the risk of further antibody-mediated platelet activation and coagulopathy, but should be considered before any intervention [31].

Treatment should not be delayed in case the result of the enzyme-linked immunoassay (ELISA) anti-PF4 autoantibodies is not available but the other features of the VITT-associated CVST exist [75]. Wolf et al showed a continuous improvement in the platelet count and spontaneous resolution within 2 weeks in a patient without immunoglobulin administration or plasmapheresis [26].

\section{Risks and Benefits of Vaccination}

Marketing of the Vaxzevria vaccine was authorized in the European Union (EU) in January 2021; and by July 4, 2021, more than 58.4 million doses had been administered in Europe [85]. After initial reported complications, on March 18, 2021, the EMA Risk Assessment Committee assumed that the vaccine was effective and safe [25]. As of June 27, 2021, 479 cases of suspected TTS with Vaxzevria were reported to EudraVigilance from EU countries; 100 of which had a fatal outcome [85]. Nearly 9 million doses on Janssen-Johnson \& Johnson vaccine were administered by May 12, 2021, with 28 reported cases of TTS [86]. A review of all available data shows that the known and potential benefits of Janssen-Johnson \& Johnson vaccine outweigh its known and potential risks [87]. Nevertheless, CVST should be considered as a very rare complication of COVID-19 vaccination [59] that can cause stroke in young adults and women of reproductive age more than others [88]. As reported, 35-50\% of COVID-19 vaccination-induced CVST cases succumb to death [26].

\section{Limitation}

The present paper reports on an evolving global phenomenon, 
and several limitations should be considered. Due to potential underreporting of side effects, the risk of adverse effects (e.g., cerebral thrombosis) following COVID-19 vaccination may be higher. Other patients may ignore related symptoms, refuse imaging or not have the training necessary to recognize alarming symptoms. A lack of information on outcome is contributing to increased vaccination hesitancy worldwide as there have been reports of life-threatening adverse events. At the time of writing this article, there is not enough information regarding the incidence of CVST-associated VITT following the second dose of adenoviral vector vaccines, as a sufficient follow-up period is not yet available. The side effect profile of all COVID-19 vaccines still needs to be investigated in future clinical trials.

\section{Conclusions}

Early diagnosis and treatment of CVST plays a fundamental role in decreasing the rate of mortality. Given the rarity of CVST after the COVID-19 vaccine, the benefit of vaccination outweighs the potential harm. According to the latest investigations, the mechanism of CVST is similar to HIT. To ensure safety, it is important not to dismiss persistent headache, seizure and other neurological symptoms in COVID-19 vaccinated individuals. Researchers and clinicians should further investigate the interactions of the vaccine with the immune system to further unravel the underlying mechanism of coagulopathy post COVID-19 vaccination.

\section{Supplementary Material}

Suppl 1. Clinical and laboratory findings of the patients with thromboembolic events after Oxford-AstraZeneca and Johnson \& Johnson COVID-19 vaccination.

\section{Acknowledgments}

None to declare.

\section{Financial Disclosure}

Authors have no relevant financial disclosure to report.

\section{Conflict of Interest}

None to declare.

\section{Author Contributions}

All authors have contributed to the theoretical formalism, designing the study, data collection, data analysis and writing the manuscript.

\section{Data Availability}

The authors declare that data supporting the findings of this study are available within the article and its supplementary file.

\section{References}

1. El Beltagi AH, Vattoth S, Abdelhady M, Ahmed I, Paksoy Y, Abou Kamar M, Alsoub H, et al. Spectrum of neuroimaging findings in COVID-19. Br J Radiol. 2021;94(1117):20200812.

2. Warkentin TE, Kaatz S. COVID-19 versus HIT hypercoagulability. Thromb Res. 2020;196:38-51.

3. Valiuddin HM, Kalajdzic A, Rosati J, Boehm K, Hill D. Update on neurological manifestations of SARS-CoV-2. West J Emerg Med. 2020;21(6):45-51.

4. Di Gennaro F, Pizzol D, Marotta C, Antunes M, Racalbuto V, Veronese N, Smith L. Coronavirus diseases (COVID-19) current status and future perspectives: a narrative review. Int J Environ Res Public Health. 2020;17(8):2690.

5. Mehta PR, Apap Mangion S, Benger M, Stanton BR, Czuprynska J, Arya R, Sztriha LK. Cerebral venous sinus thrombosis and thrombocytopenia after COVID-19 vaccination - A report of two UK cases. Brain Behav Immun. 2021;95:514-517.

6. Valderrama EV, Humbert K, Lord A, Frontera J, Yaghi S. Severe Acute Respiratory Syndrome Coronavirus 2 Infection and Ischemic Stroke. Stroke. 2020;51(7):e124e127.

7. Song P, Li W, Xie J, Hou Y, You C. Cytokine storm induced by SARS-CoV-2. Clin Chim Acta. 2020;509:280287.

8. von Hundelshausen P, Lorenz R, Siess W, Weber C. Vaccine-induced immune thrombotic thrombocytopenia (VITT): targeting pathomechanisms with Bruton Tyrosine Kinase inhibitors. Thromb Haemost. 2021.

9. Kantarcioglu B, Iqbal O, Walenga JM, Lewis B, Lewis J, Carter CA, Singh M, et al. An Update on the Pathogenesis of COVID-19 and the Reportedly Rare Thrombotic Events Following Vaccination. Clin Appl Thromb Hemost. 2021;27:10760296211021498.

10. Marcucci R, Marietta M. Vaccine-induced thrombotic thrombocytopenia: the elusive link between thrombosis and adenovirus-based SARS-CoV-2 vaccines. Intern Emerg Med. 2021;16(5):1113-1119.

11. Bussel J, Connors J, Cines D. Thrombosis with thrombocytopenia syndrome (also termed vaccine-induced thrombotic thrombocytopenia. Version 1.2. American Society of Hematology. April 25, 2021.

12. Pai M, Grill A, Ivers N. Vaccine-induced prothrombotic immune thrombocytopenia VIPIT following AstraZeneca COVID-19 vaccination. Science Briefs of the Ontario COVID-19 Science Advisory Table. 2021;1(17).

13. Hernandez AF, Calina D, Poulas K, Docea AO, Tsatsakis AM. Safety of COVID-19 vaccines administered in the EU: Should we be concerned? Toxicol Rep. 2021;8:871879. 
14. See I, Su JR, Lale A, Woo EJ, Guh AY, Shimabukuro TT, Streiff MB, et al. US case reports of cerebral venous sinus thrombosis with thrombocytopenia after Ad26. COV2.S vaccination, March 2 to April 21, 2021. JAMA. 2021;325(24):2448-2456.

15. Blauenfeldt RA, Kristensen SR, Ernstsen SL, Kristensen $\mathrm{CCH}$, Simonsen CZ, Hvas AM. Thrombocytopenia with acute ischemic stroke and bleeding in a patient newly vaccinated with an adenoviral vector-based COVID-19 vaccine. J Thromb Haemost. 2021;19(7):1771-1775.

16. Schultz NH, Sorvoll IH, Michelsen AE, Munthe LA, Lund-Johansen F, Ahlen MT, Wiedmann M, et al. Thrombosis and Thrombocytopenia after ChAdOx1 nCoV-19 Vaccination. N Engl J Med. 2021;384(22):2124-2130.

17. Bayas A, Menacher M, Christ M, Behrens L, Rank A, Naumann M. Bilateral superior ophthalmic vein thrombosis, ischaemic stroke, and immune thrombocytopenia after ChAdOx1 nCoV-19 vaccination. Lancet. 2021;397(10285):e11.

18. Bjornstad-Tuveng TH, Rudjord A, Anker P. Fatal cerebral haemorrhage after COVID-19 vaccine. Tidsskr Nor Laegeforen. 2021;141.

19. Castelli GP, Pognani C, Sozzi C, Franchini M, Vivona L. Cerebral venous sinus thrombosis associated with thrombocytopenia post-vaccination for COVID-19. Crit Care. 2021;25(1):137.

20. D'Agostino V, Caranci F, Negro A, Piscitelli V, Tuccillo B, Fasano F, Sirabella G, et al. A rare case of cerebral venous thrombosis and disseminated intravascular coagulation temporally associated to the COVID-19 vaccine administration. J Pers Med. 2021;11(4):285.

21. Franchini M, Testa S, Pezzo M, Glingani C, Caruso B, Terenziani I, Pognani C, et al. Cerebral venous thrombosis and thrombocytopenia post-COVID-19 vaccination. Thromb Res. 2021;202:182-183.

22. Greinacher A, Selleng K, Warkentin TE. Autoimmune heparin-induced thrombocytopenia. J Thromb Haemost. 2017;15(11):2099-2114.

23. Muir KL, Kallam A, Koepsell SA, Gundabolu K. Thrombotic thrombocytopenia after Ad26.COV2.S vaccination. N Engl J Med. 2021;384(20):1964-1965.

24. Scully M, Singh D, Lown R, Poles A, Solomon T, Levi M, Goldblatt D, et al. Pathologic antibodies to platelet factor 4 after ChAdOx1 nCoV-19 vaccination. N Engl J Med. 2021;384(23):2202-2211.

25. Tobaiqy M, Elkout H, MacLure K. Analysis of thrombotic adverse reactions of COVID-19 AstraZeneca vaccine reported to EudraVigilance database. Vaccines (Basel). 2021;9(4):393.

26. Wolf ME, Luz B, Niehaus L, Bhogal P, Bazner H, Henkes $\mathrm{H}$. Thrombocytopenia and intracranial venous sinus thrombosis after "COVID-19 Vaccine AstraZeneca" exposure. J Clin Med. 2021;10(8):1599.

27. Aladdin Y, Algahtani H, Shirah B. Vaccine-Induced Immune Thrombotic Thrombocytopenia with disseminated intravascular coagulation and death following the ChAdOx1 nCoV-19 vaccine. J Stroke Cerebrovasc Dis. 2021;30(9):105938.

28. Bano F, Badugama B, Chandra D. Thrombosis and thrombocytopaenia after $\mathrm{ChAdOx} 1 \mathrm{nCoV}-19$ vaccination: a single UK centre experience. BMJ Case Rep. 2021;14(7):e243894.

29. Clark RT, Johnson L, Billotti J, Foulds G, Ketels T, Heard K, Calvello Hynes E. Early outcomes of bivalirudin therapy for thrombotic thrombocytopenia and cerebral venous sinus thrombosis after Ad26.COV2.S vaccination. Ann Emerg Med. 2021.

30. Dutta A, Ghosh R, Bhattacharya D, Bhat S, Ray A, Pandit A, Das S, et al. Anti-PF4 antibody negative cerebral venous sinus thrombosis without thrombocytopenia following immunization with COVID-19 vaccine in an elderly non-comorbid Indian male, managed with conventional heparin-warfarin based anticoagulation. Diabetes Metab Syndr. 2021;15(4):102184.

31. Gessler F, Schmitz AK, Dubinski D, Bernstock JD, Lehmann F, Won SY, Wittstock M, et al. Neurosurgical considerations regarding decompressive craniectomy for intracerebral hemorrhage after SARS-CoV-2-vaccination in vaccine induced thrombotic thrombocytopenia-VITT. J Clin Med. 2021;10(13):2777.

32. Guan CY, Tsai SH, Fan JS, Lin YK, Kao CC. A rare case of a middle-age Asian male with cerebral venous thrombosis after COVID-19 AstraZeneca vaccination. Am J Emerg Med. 2021.

33. Suresh P, Petchey W. ChAdOx1 nCOV-19 vaccine-induced immune thrombotic thrombocytopenia and cerebral venous sinus thrombosis (CVST). BMJ Case Rep. 2021;14(6):e243931.

34. Tolboll Sorensen AL, Rolland M, Hartmann J, Harboe ZB, Roed C, Jensen TO, Kolte L, et al. A case of thrombocytopenia and multiple thromboses after vaccination with ChAdOx1 nCoV-19 against SARS-CoV-2. Blood Adv. 2021;5(12):2569-2574.

35. Walter U, Fuchs M, Grossmann A, Walter M, Thiele T, Storch A, Wittstock M. Adenovirus-vectored COVID-19 vaccine-induced immune thrombosis of carotid artery: a case report. Neurology. 2021.

36. AHA. CVST and blood clots potentially related to the J\&J COVID-19 vaccine: know the symptoms. April 15, 2021. Available from: https://newsroom.heart.org/news/ cvst-and-blood-clots-potentially-related-to-the-j-j-covid19-vaccine-know-the-symptoms?preview $=885 \mathrm{~d}$.

37. Fatima N, Saqqur M, Qamar F, Shaukat S, Shuaib A. Impact of COVID-19 on neurological manifestations: an overview of stroke presentation in pandemic. Neurol Sci. 2020;41(10):2675-2679.

38. Shakibajahromi B, Borhani-Haghighi A, Haseli S, Mowla A. Cerebral venous sinus thrombosis might be underdiagnosed in the COVID-19 era. eNeurologicalSci. 2020;20:100256.

39. Klok FA, Kruip M, van der Meer NJM, Arbous MS, Gommers D, Kant KM, Kaptein FHJ, et al. Incidence of thrombotic complications in critically ill ICU patients with COVID-19. Thromb Res. 2020;191:145-147.

40. Kaur I, Vyas C, Mughal M, Gandhi H, Du D. Cerebral venous sinus thrombosis in COVID-19: an unusual presentation. Cureus. 2021;13(3):e13767.

41. Li Y, Li M, Wang M, Zhou Y, Chang J, Xian Y, Wang 
D, et al. Acute cerebrovascular disease following COVID-19: a single center, retrospective, observational study. Stroke Vasc Neurol. 2020;5(3):279-284.

42. Vahabizad F, Sharifian Dorche M, Mohammadi P, Khatibi K, Mowla A. COVID-19-related acute ischemic stroke in young adults: what is the optimal antithrombotic regimen for secondary prevention? J Neurol Res. 2020;10(5):203206.

43. Shahjouei S, Anyaehie M, Koza E, Tsivgoulis G, Naderi S, Mowla A, Avula V, et al. SARS-CoV-2 is a culprit for some, but not all acute ischemic strokes: a report from the multinational COVID-19 stroke study group. J Clin Med. 2021;10(5):931.

44. Sharifian-Dorche M, Huot P, Osherov M, Wen D, Saveriano A, Giacomini PS, Antel JP, et al. Neurological complications of coronavirus infection; a comparative review and lessons learned during the COVID-19 pandemic. J Neurol Sci. 2020;417:117085.

45. Mowla A, Sizdahkhani S, Sharifian-Dorche M, Selvan P, Emanuel BA, Tenser MS, Amar AP, et al. Unusual pattern of arterial macrothrombosis causing stroke in a young adult recovered from COVID-19. J Stroke Cerebrovasc Dis. 2020;29(12):105353.

46. Shahjouei S, Naderi S, Li J, Khan A, Chaudhary D, Farahmand G, Male S, et al. Risk of stroke in hospitalized SARS-CoV-2 infected patients: a multinational study. EBioMedicine. 2020;59:102939.

47. Dmytriw AA, Phan K, Schirmer C, Settecase F, Heran MKS, Efendizade A, Kuhn AL, et al. Ischaemic stroke associated with COVID-19 and racial outcome disparity in North America. J Neurol Neurosurg Psychiatry. 2020;91(12):1362-1364.

48. Hamidianjahromi A, Mowla A. Re: The choice of intravenous thrombolysis for Acute Ischemic Stroke under COVID-19 infection. Clin Neurol Neurosurg. 2021;202:106501.

49. Mowla A. Stroke care during the COVID-19 pandemic; a global challenge. Iran J Med Sci. 2020;45(5):323-324.

50. Sharifian-Dorche M, Mowla A. Neurological care during the pandemic; a global challenge. Journal of Experimental and Clinical Neurosciences. 2020;7(2).

51. Shahjouei S, Tsivgoulis G, Farahmand G, Koza E, Mowla A, Vafaei Sadr A, Kia A, et al. SARS-CoV-2 and stroke characteristics: a report from the multinational COVID-19 stroke study group. Stroke. 2021;52(5):e117-e130.

52. Hamidianjahromi A, Mowla A. Letter regarding 'Neurointervention for emergent large vessel occlusion strokes during the COVID-19 pandemic'. J Neurointerv Surg. 2021;13(9):e20.

53. Jafari Khaljiri H, Jamalkhah M, Amini Harandi A, Pakdaman H, Moradi M, Mowla A. Comprehensive review on neuro-COVID-19 pathophysiology and clinical consequences. Neurotox Res. 2021;39(5):1613-1629.

54. Dmytriw AA, Dibas M, Schirmer CM, Settecase F, Heran MKS, Efendizade A, Kuhn AL, et al. Age and acute ischemic stroke outcome in north american patients with COVID-19. J Am Heart Assoc. 2021;10(14):e021046.

55. Ostovan VR, Foroughi R, Rostami M, Almasi-Dooghaee M, Esmaili M, Bidaki AA, Behzadi Z, et al. Cerebral ve- nous sinus thrombosis associated with COVID-19: a case series and literature review. J Neurol. 2021.

56. Luo Y, Tian X, Wang X. Diagnosis and Treatment of Cerebral Venous Thrombosis: A Review. Front Aging Neurosci. 2018;10:2.

57. Smadja DM, Yue QY, Chocron R, Sanchez O, Lillo-Le Louet A. Vaccination against COVID-19: insight from arterial and venous thrombosis occurrence using data from VigiBase. Eur Respir J. 2021;58(1):2100956.

58. Shakibajahromi B, Haghighi AB, Salehi A, Vardanjani HM, Ghaedian M, Safari A, Mowla A. Clinical and radiological characteristics and predictors of outcome of cerebral venous sinus thrombosis, a hospital-based study. Acta Neurol Belg. 2020;120(4):845-852.

59. Mowla A, Shakibajahromi B, Shahjouei S, BorhaniHaghighi A, Rahimian N, Baharvahdat H, Naderi S, et al. Cerebral venous sinus thrombosis associated with SARS-CoV-2; a multinational case series. J Neurol Sci. 2020;419:117183.

60. Shakibajahromi B, Borhani-Haghighi A, Vardanjani HM, Ghaedian M, Feiz F, Safari A, et al. Isolated headache and its association with characteristics and outcome of cerebral venous sinus thrombosis. Cephalalgia Reports. 2020;3:2515816320919961.

61. Shakibajahromi B, Borhani-Haghighi A, Ghaedian M, Feiz F, Molavi Vardanjani H, Safari A, Salehi A, et al. Early, delayed, and expanded intracranial hemorrhage in cerebral venous thrombosis. Acta Neurol Scand. 2019;140(6):435-442.

62. Stam J. Thrombosis of the cerebral veins and sinuses. N Engl J Med. 2005;352(17):1791-1798.

63. Yavasoglu I. Vaccination and thrombotic thrombocytopenic purpura. Turk J Haematol. 2020;37(3):218-219.

64. Brown RC, Blecher TE, French EA, Toghill PJ. Thrombotic thrombocytopenic purpura after influenza vaccination. Br Med J. 1973;2(5861):303.

65. Kojima Y, Ohashi H, Nakamura T, Nakamura H, Yamamoto H, Miyata Y, Iida H, et al. Acute thrombotic thrombocytopenic purpura after pneumococcal vaccination. Blood Coagul Fibrinolysis. 2014;25(5):512-514.

66. Hermann R, Pfeil A, Busch M, Kettner C, Kretzschmar D, Hansch A, La Rosee P, et al. [Very severe thrombotic thrombocytopenic purpura (TTP) after H1N1 vaccination]. Med Klin (Munich). 2010;105(9):663-668.

67. Kadikoylu G, Yavasoglu I, Bolaman Z. Rabies vaccineassociated thrombotic thrombocytopenic purpura. Transfus Med. 2014;24(6):428-429.

68. Ramakrishnan N, Parker LP. Thrombotic thrombocytopenic purpura following influenza vaccination - a brief case report. Conn Med. 1998;62(10):587-588.

69. Chan BT, Bobos P, Odutayo A, Pai M. Meta-Analysis of Risk of Vaccine-Induced Immune Thrombotic Thrombocytopenia Following ChAdOx1-S Recombinant Vaccine. medRxiv. 2021.

70. Cari L, Fiore P, Naghavi Alhosseini M, Sava G, Nocentini G. Blood clots and bleeding events following BNT162b2 and ChAdOx1 nCoV-19 vaccine: An analysis of European data. J Autoimmun. 2021;122:102685.

71. Warkentin TE, Makris M, Jay RM, Kelton JG. A spon- 
taneous prothrombotic disorder resembling heparin-induced thrombocytopenia. Am J Med. 2008;121(7):632636.

72. Franchini M, Liumbruno GM, Pezzo M. COVID-19 vaccine-associated immune thrombosis and thrombocytopenia (VITT): Diagnostic and therapeutic recommendations for a new syndrome. Eur J Haematol. 2021;107(2):173180.

73. Hogan M, Berger JS. Heparin-induced thrombocytopenia (HIT): Review of incidence, diagnosis, and management. Vasc Med. 2020;25(2):160-173.

74. Smythe MA, Koerber JM, Mattson JC. The incidence of recognized heparin-induced thrombocytopenia in a large, tertiary care teaching hospital. Chest. 2007;131(6):16441649.

75. Dotan A, Shoenfeld Y. Perspectives on vaccine induced thrombotic thrombocytopenia. J Autoimmun. 2021;121:102663.

76. Lundstrom K, Barh D, Uhal BD, Takayama K, Aljabali AAA, Abd El-Aziz TM, Lal A, et al. COVID-19 vaccines and thrombosis-roadblock or dead-end street? Biomolecules. 2021;11(7):1020.

77. Mastellos DC, Skendros P, Lambris JD. Is complement the culprit behind COVID-19 vaccine-related adverse reactions? J Clin Invest. 2021;131(11):e151092.

78. Kowarz E, Krutzke L, Reis J, Bracharz S, Kochanek S, Marschalek R. "Vaccine-Induced COVID-19 Mimicry" Syndrome: Splice reactions within the SARS-CoV-2 Spike open reading frame result in Spike protein variants that may cause thromboembolic events in patients immunized with vector-based vaccines. 2021

79. Stone D, Liu Y, Shayakhmetov D, Li ZY, Ni S, Lieber A. Adenovirus-platelet interaction in blood causes virus sequestration to the reticuloendothelial system of the liver. J Virol. 2007;81(9):4866-4871.

80. Marchetti M, Zermatten MG, Bertaggia Calderara D, Ali- otta A, Alberio L. Heparin-Induced thrombocytopenia: a review of new concepts in pathogenesis, diagnosis, and management. J Clin Med. 2021;10(4):683.

81. CDC. Cases of Cerebral venous sinus thrombosis with thrombocytopenia after receipt of the Johnson \& Johnson COVID-19 vaccine. April 13, 2021. Available from: https://emergency.cdc.gov/han/2021/han00442.asp.

82. Greinacher A, Thiele T, Warkentin TE, Weisser K, Kyrle $\mathrm{P}$, Eichinger S. A prothrombotic thrombocytopenic disorder resembling heparin-induced thrombocytopenia following coronavirus-19 vaccination. 2021.

83. Warkentin TE, Pai M, Linkins LA. Direct oral anticoagulants for treatment of HIT: update of Hamilton experience and literature review. Blood. 2017;130(9):1104-1113.

84. Chen PW, Tsai ZY, Chao TH, Li YH, Hou CJ, Liu PY. Addressing vaccine-induced immune thrombotic thrombocytopenia (VITT) following COVID-19 vaccination: a mini-review of practical strategies. Acta Cardiol Sin. 2021;37(4):355-364.

85. European Medicines Agency. Safety update, July 14, 2021. Available from: https://www.ema.europa.eu/en/ medicines/human/EPAR/vaxzevria-previously-covid19-vaccine-astrazeneca\#safety-updates-section.

86. Organization WH. Statement of the COVID-19 subcommittee of the WHO Global Advisory Committee on Vaccine Safety (GACVS) on safety signals related to the Johnson \& Johnson/Janssen COVID-19 vaccine. May 12, 2021. Available from: https://www.who.int/news/ item/19-05-2021-statement-gacvs-safety-johnson-johnson-janssen-covid-19-vaccine.

87. Prevention CfDCa. CDC Recommends Use of Johnson \& Johnson's Janssen COVID-19 Vaccine Resume. July 13, 2021. Available from: https://www.cdc.gov/ coronavirus/2019-ncov/vaccines/safety/JJUpdate.html.

88. Hunter PR. Thrombosis after COVID-19 vaccination. BMJ. 2021;373:n958. 